\title{
Performance Assessment of PAPR in OFDM System using Single Carrier - FDMA
}

\author{
Arjun Solanki \\ Research Scholar \\ Medicaps Ins. Of Technology \& Management \\ Dept. of Electronics \& Communication
}

\author{
Ratna Gour \\ Sr. Assistant Professor \\ Medicaps Ins. Of Technology \& Management \\ Dept. of Electronics \& Communication
}

\begin{abstract}
Orthogonal frequency division multiplexing (OFDM) is a promising multicarrier modulation technique for high data rate communication. Unless, OFDM faces the peak-toaverage-power ratio (PAPR) problem that is a major drawback of multicarrier transmission system which leads to power inefficiency of high power amplifier (HPA) and also demands the large dynamic range of digital to analog converter (DAC) at the transmitter. A number of schemes have been proposed to reduce the PAPR problem. The Discrete Fourier Transform (DFT) Spreading is one of the scheme to reduce the PAPR Problem in OFDM system. This paper presents the DFT spreading technique to reduce the PAPR problem. The PAPR reduction capability of this technique is demonstrated by presenting simulation results of PAPR
\end{abstract}

\section{Keywords}

Interleaved frequency division multiple access (IFDMA), Localized frequency division multiple access (LFDMA), Orthogonal frequency division multiplexing (OFDM), Peak to average power ratio (PAPR).

\section{INTRODUCTION}

Orthogonal Frequency Division Multiplexing (OFDM), which is one of multi-carrier modulation (MCM) techniques, offers considerable bandwidth efficiency, high spectral efficiency, multipath delay spread tolerance, power efficiency and immunity to the frequency selective fading channels. OFDM has been chosen for high data rate communications and has been widely deployed in many wireless communication standards such as Digital Video Broadcasting (DVB) and mobile worldwide interoperability for microwave access (mobile WiMAX) based on OFDM access technology [1]. Orthogonal means numbers of subcarriers are overlapped but they are independent with each other at any specified time interval, these subcarrier should be densely spaced Theoretically. PAPR reduction schemes can be classified according to several criteria. First, the PAPR schemes can be categorized as multiplicative and additive schemes with respect to the computational operation in the frequency domain. Selected mapping (SLM) and partial transmit sequences (PTS) are examples of the multiplicative scheme because the phase sequences are multiplied by the input symbol vectors in the frequency domain. On the other hand, tone reservation (TR), peak canceling, and clipping are additive schemes, because peak reduction vectors are added to the input symbol vector. Second, the PAPR reduction schemes can be also categorized according to whether they are probabilistic or deterministic. Deterministic schemes, such as clipping and peak canceling, strictly limit the PAPR of the OFDM signals below a given threshold level. Probabilistic Schemes are statistically improve, the characteristics of the
PAPR distribution of the OFDM signals avoiding signal distortion. SLM and PTS are examples of the probabilistic scheme because several candidate signals are generated and that which has the minimum PAPR is selected for transmission [2].

Besides the PAPR reduction schemes, the single carrier frequency division multiple access (SC-FDMA) scheme has been proposed for alleviating the PAPR problem in uplink transmission. The SC-FDMA is a adopted multiple access scheme for uplink transmission in the long term evolution (LTE) of cellular systems by the third generation partnership project (3GPP). It is clear that the PAPR of SC-FDMA is lower than that of OFDMA, because SC-FDMA transmits the input symbols sequentially using a single carrier, while OFDMA theoretically transmits the input symbols in parallel.

\section{SYSTEM MODEL}

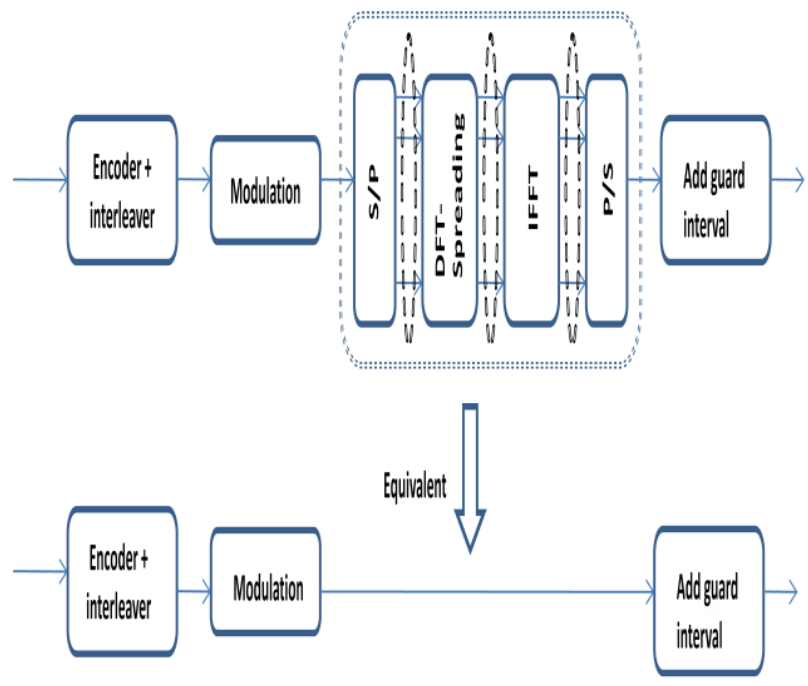

Fig 1: Equivalent of OFDMA system with DFT-spreading code to a single-carrier system.

Before discussing the DFT-spreading technique, let us consider OFDMA (Orthogonal Frequency- Division Multiple Access) system. Figure 1 shows, suppose that DFT of the same size as IFFT is used as a (spreading) code. Then, the OFDMA system becomes equivalent to the Single Carrier FDMA (SC-FDMA) system because the DFT and IDFT operations virtually cancel each other. In this case, the transmit signal will have the same PAPR as in a single-carrier system [3].

In DFT spreading, there are two different approaches of 
assigning subcarriers among users: DFDMA (Distributed FDMA) and LFDMA (Localized FDMA) [4].
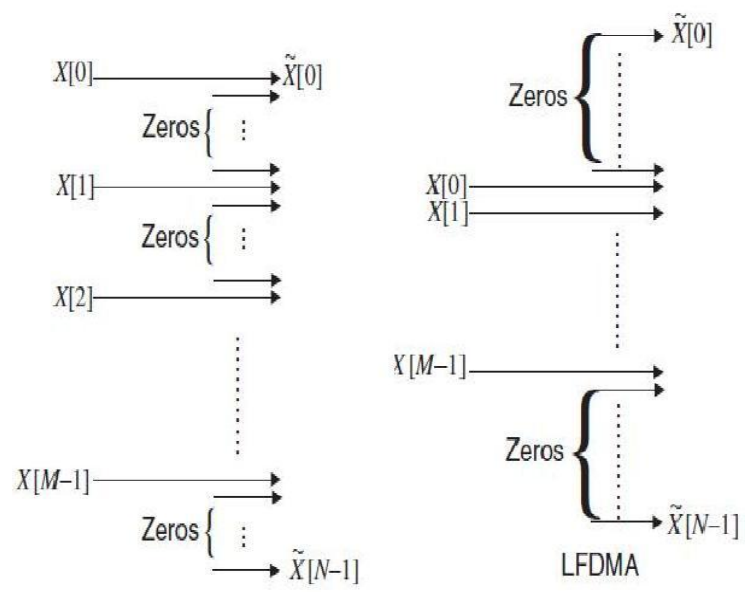

DFDMA

Fig 2: subcarrier mapping for uplink transmission in DFDMA and LFDMA systems. Shows DFDMA distributes M DFT outputs over all $\mathrm{N}$ subcarriers with zeros filled in unused subcarriers i.e.(N-M), whereas LFDMA allocates DFT outputs to $\mathrm{M}$ consecutive subcarriers in $\mathrm{N}$ subcarrier. When DFDMA distributes DFT outputs with equidistance N/M $=S$, it is referred to as IFDMA (Interleaved FDMA) where $\mathrm{S}$ is called the bandwidth spreading factor.
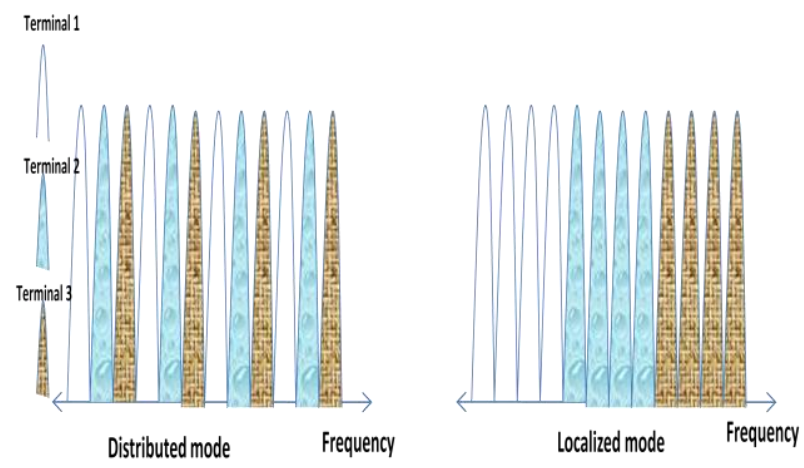

Fig 3: subcarrier assisgnment in DFDMA and LFDMA system, when $\mathrm{N}=12, \mathrm{M}=4, \mathrm{~S}=3$.
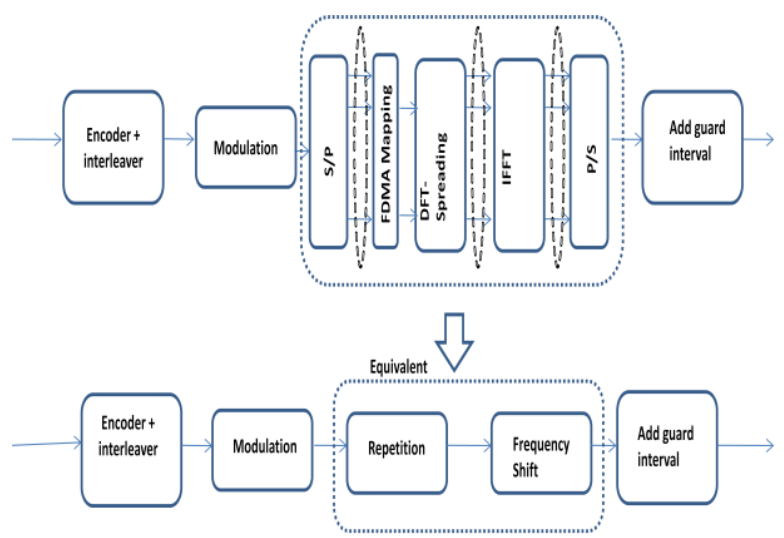

Fig 4: uplink transmitter for DFT-Spreading technique.

\subsection{SYSTEM MODEL DESCRIPTION}

In DFT-Spreading transmitter model we are using an FDMA mapping to adderss the subcarrier allocation method and DFT-Sprading code, then the equivalent system becomes single carrier frequecncy division multiple access (SCFDMA), so to understand the repetition and frequency shift in the equivalent transmitter model we will go through the mathmatical description [6], here the input data $x(m)$ is the DFT-Spread to generate $\mathrm{x}(\mathrm{i})$ so equation is, Here the input data $x[M]$ is DFT-spread to generate $X[i]$ and then allocated as

$\tilde{X}[k]=\left\{\begin{array}{rr}X[k / S] & k=S * m 1 \\ 0 & \text { else }\end{array}\right.$

The IFFT output sequence $\check{x}[n]$ with $\mathrm{n}=\mathrm{M} * \mathrm{~s}+\mathrm{m}$ for $\mathrm{s}=0,1$, $2 \ldots \ldots . \mathrm{S}-1$ and $\mathrm{m}=0,1,2 \ldots . \mathrm{M}-1$ can be expressed as

$\check{x}[n]=\frac{1}{N} \sum_{k=0}^{N-1} \breve{X}[k] e^{j 2 \pi \frac{n k}{n}}(1.2)$

$\check{x}[n]=\frac{1}{S} \frac{1}{M} \sum_{m 1=0}^{M-1} \breve{X}[m 1] e^{j 2 \pi \frac{n m 1}{M}}$

$\breve{x}[n]=\frac{1}{S} \frac{1}{M} \sum_{m 1=0}^{M-1} \breve{X}[m 1] e^{j 2 \pi \frac{M s+m * m 1}{M}}$

$\breve{X}[n]=\frac{1}{S}\left(\frac{1}{M} \sum_{m 1=0}^{M-1} \breve{X}[m 1] e^{j 2 \pi \frac{m * m 1}{M}}\right)$

$\breve{x}[n]=\frac{1}{S} X[m]$

Which is the repetition of the original input signal $X[\mathrm{~m}]$ scaled by $1 / \mathrm{S}$ in the time domain, in the IFDMA where subcarrier mapping starts with the rth subcarrier $r=0,1$, $2 \ldots \ldots \ldots \ldots$... 1 , the DFT spread symbol can be expressed as

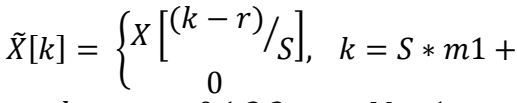

$r$ where $m=0,1,2,3, \ldots \ldots, M-1$

(1.7)

Then the corresponding IFFT output sequence $\breve{x}[n]$ is given by

$\check{x}[n]=\breve{x}[M s+m]$

$\check{x}[n]=\frac{1}{N} \sum_{k=0}^{N-1} \breve{X}[k] e^{j 2 \pi \frac{n k}{n}}$

$\breve{x}[n]=\frac{1}{S} \frac{1}{M} \sum_{m 1=0}^{M-1} \breve{X}[m 1] e^{j 2 \pi\left(\frac{n m 1}{M}+\frac{n r}{M}\right)}$

$\check{\chi}[n]=\frac{1}{S} \frac{1}{M} \sum_{m 1=0}^{M-1} \breve{X}[m 1] e^{j 2 \pi \frac{M S+m * m 1}{M}} * e^{j 2 \pi \frac{n r}{N}}$

(1.11)

$\breve{x}[n]=\frac{1}{S} e^{j 2 \pi \frac{n r}{N}} X[m]$

(1.12)

Compared with equation with (1.4) one can see that the frequency shift of subcarrier allocation starting point by $r$ subcarrier results in the phase rotation of $e^{j 2 \pi^{n r} / N}$ in IFDMA. 
In the DFT-spreading scheme for LFDMA the IFFT input signal $\breve{x}[k]$ at the transmitter can be expressed as

$$
\check{X}[k]=\left\{\begin{array}{l}
X[k], \quad k=0,1,2, \ldots \ldots \ldots \ldots . M-1 \\
0 \quad, k=M, M+1, M+2 \ldots N-1
\end{array}\right.
$$

The IFFT outputs sequence $\breve{x}[n]$ with $\mathrm{n}=\mathrm{S} * \mathrm{~m}+\mathrm{s}$ for $\mathrm{S}=0,1$, 2 ........S-1 can be expressed as follows

$\check{x}[n]=\check{x}[S m+s]$

$\check{x}[n]=\frac{1}{N} \sum_{k=0}^{N-1} \breve{X}[k] e^{j 2 \pi \frac{n k}{n}}$

(1.15)

$\check{x}[n]=\frac{1}{S} \frac{1}{M} \sum_{k=0}^{N-1} \breve{X}[k] e^{j 2 \pi \frac{S M+s * k}{S M}}$

(1.16)

For $\mathrm{s}=0$,

$$
\check{x}[n]=\breve{x}[S m]
$$

$\check{x}[n]=\frac{1}{S} \frac{1}{M} \sum_{k=0}^{M-1} \breve{X}[k] e^{j 2 \pi \frac{S m k}{S M}}$

$\check{x}[n]=\frac{1}{S} \frac{1}{M} \sum_{k=0}^{M-1} \breve{X}[k] e^{j 2 \pi \frac{m k}{M}}$

(1.19)

$\check{x}[n]=\frac{1}{S} X[m]$

For $\mathrm{s} \neq 0$

$$
\breve{X}[k]=\sum_{p=0}^{M-1} x[p] e^{-j 2 \pi \frac{p k}{N}}
$$

$\check{x}[n]=\check{x}[S m+s]$

(1.22)

$$
\check{x}[n]=\frac{1}{S}\left(1-e^{j 2 \pi \frac{s}{s}}\right) * \frac{1}{M} \sum_{p=0}^{M-1} \quad \frac{x[p]}{1-\left(e^{-j 2 \pi \frac{(m-p)}{M}+\frac{s}{S M}}\right)}
$$

$$
\begin{aligned}
& \check{x}[n]=\frac{1}{S} e^{j 2 \pi \frac{(M-1) s-S m}{S M}} \sum_{p=0}^{M-1} \\
& \frac{\sin \pi \frac{s}{S}}{(\mathrm{M}) \sin \pi\left(\frac{(S M+s)}{S M}-\frac{\pi p}{M}\right)} e^{j \pi \frac{p}{M}} x[p]
\end{aligned}
$$

From equation $1.23 \& 1.24$, it can be seen that the time domain LFDMA signal becomes the 1/S scaled copies of the input sequence at the multiples of $\mathrm{S}$ in the time domain. The values in between are obtained by summing all the input sequences with the different complex weight factor.

\section{SIMULATION RESULT AND}

\section{DISCUSSION}

All the simulation has been done in MATLAB, as we know there are two types of channel allocations method in DFTSpreading technique. In the following simulation results we compared different allocatio schemes like OFDMA,LFDMA,IFDMA and their performance againest
PAPR. The simulation results helps in evluating the PAPR performance using DFT-Spreading and in presence of different modulation techniques like BPSK,QPSK,16QAM,64-QAM.

As comparing different allocation technique with different modulation techniques, means comparing fig 5, fig6, fig7, fig8, we can easily justify that the performance of 16-QAM modulation technique is better and the value of IFDMA is $3.8 \mathrm{~dB}$, for LFDMA $8.3 \mathrm{~dB}$, and OFDMA $10.3 \mathrm{~dB}$. So IFDMA is performing better as compared to the OFDMA and LFDMA, and also on the basis of DFT-Spreading the performance of IFDMA and LFDMA I is better than OFDMA in uplink transmission.

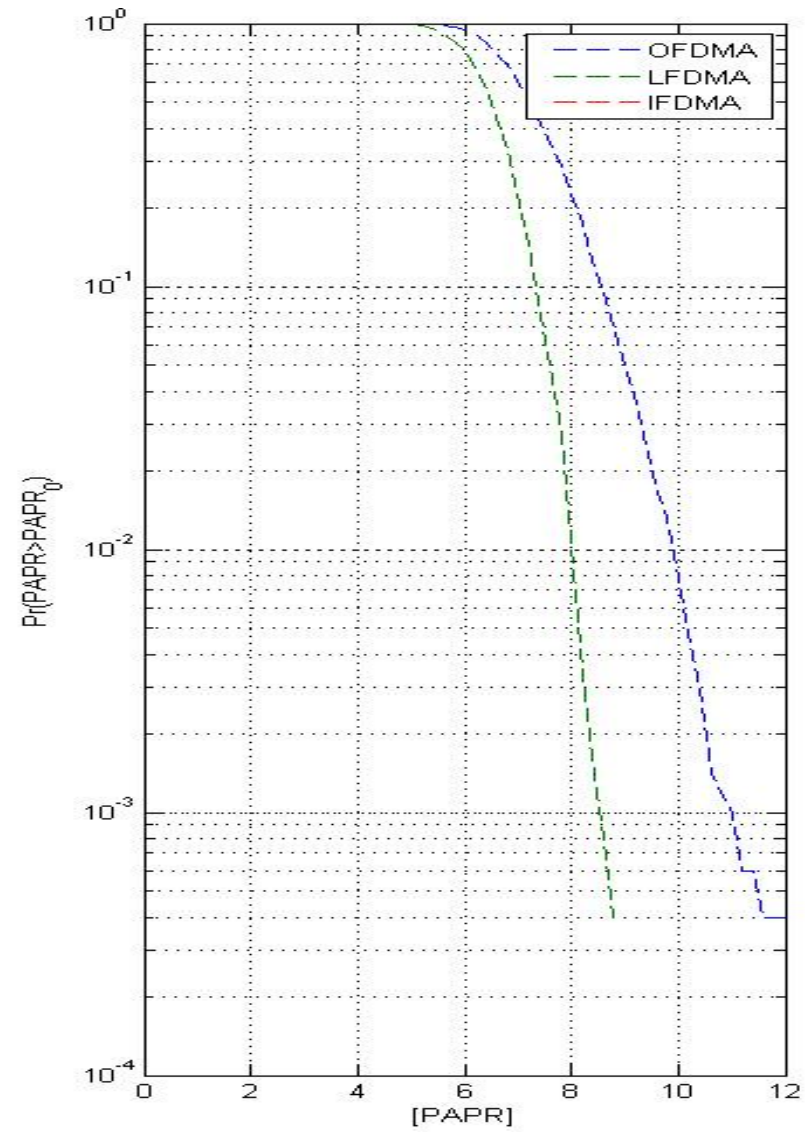

Fig 5 : Simulation with BPSK. 


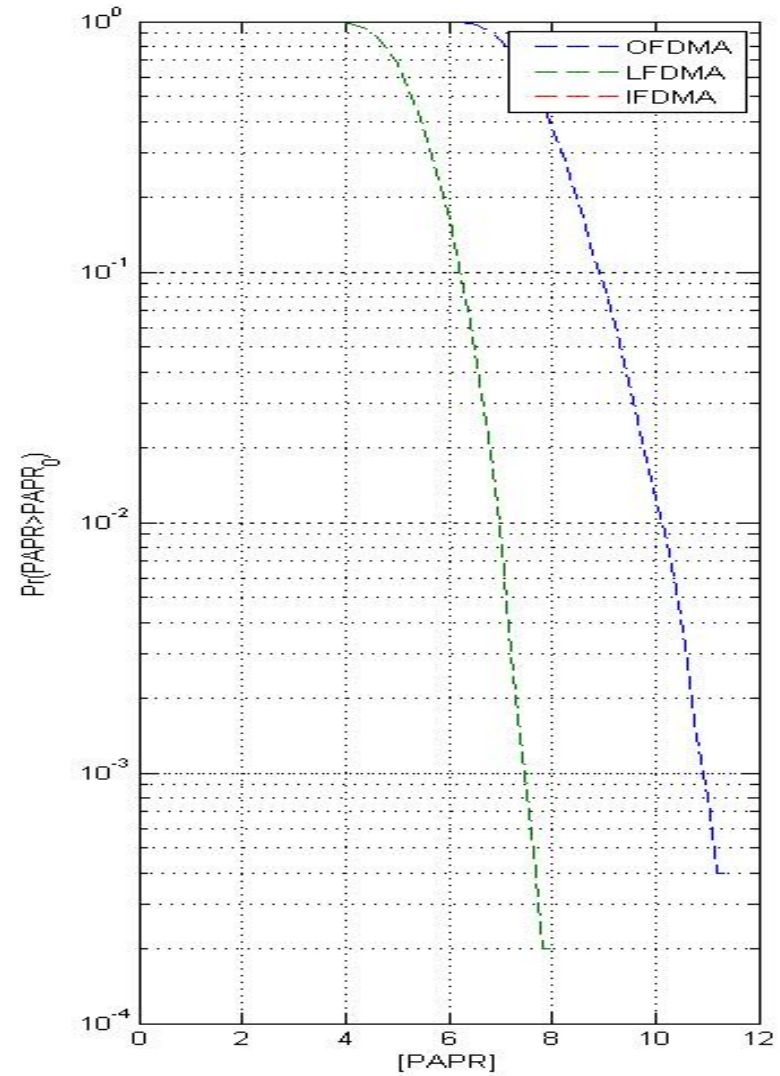

Fig 6 : Simulation with QPSK.

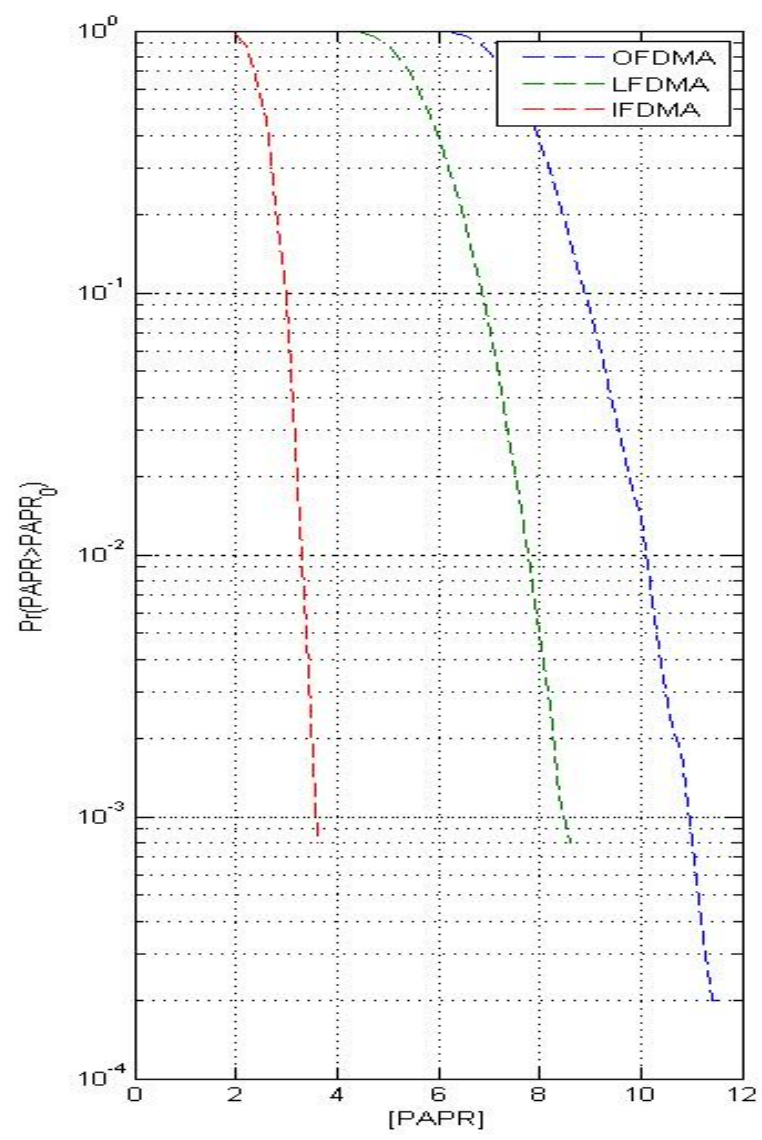

Fig 7: Simulation with 16-QAM.

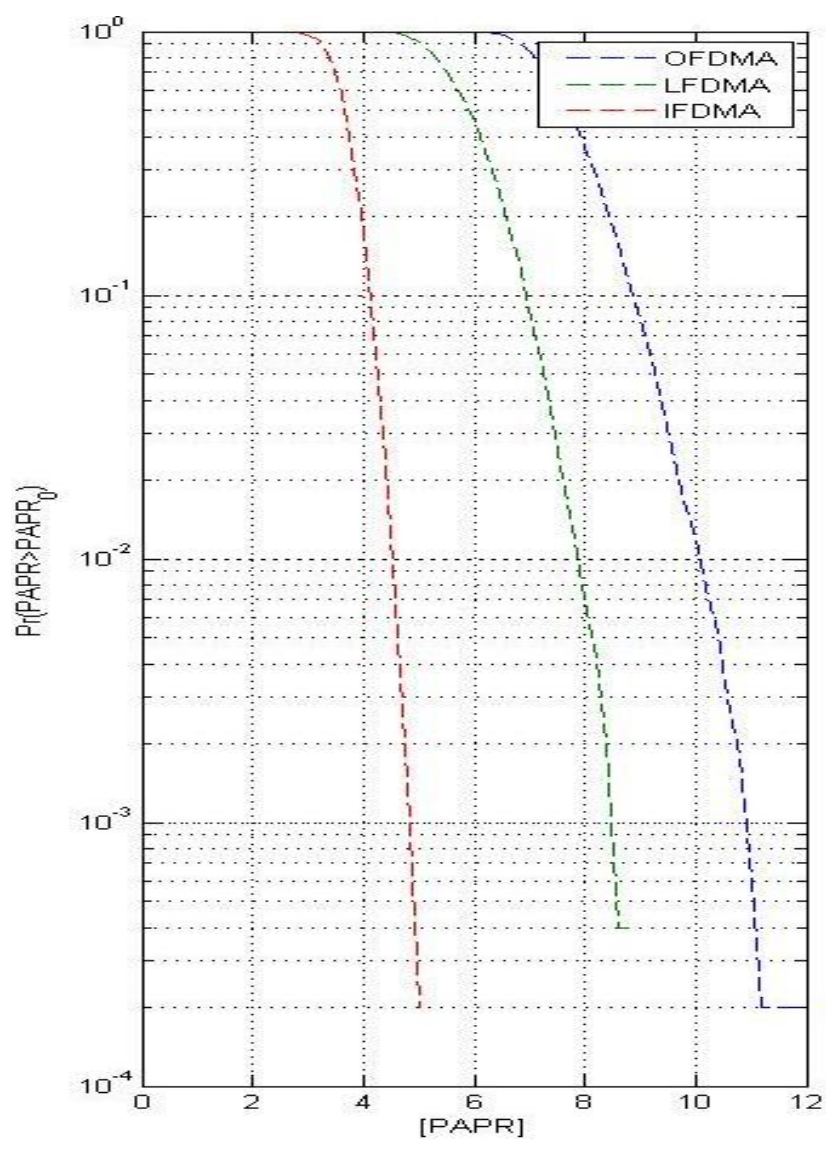

Fig 8: Simulation with 64-QAM.

Table 1. performance comparison of allocation methods

\begin{tabular}{|l|l|l|l|l|}
\hline $\begin{array}{l}\text { Allocation } \\
\text { Method }\end{array}$ & $\begin{array}{l}\text { BPSK } \\
(\mathbf{d B})\end{array}$ & $\begin{array}{l}\text { QPSK } \\
(\mathbf{d B})\end{array}$ & $\begin{array}{l}\text { 16-QAM } \\
(\mathbf{d B})\end{array}$ & $\begin{array}{l}\text { 64- } \\
\text { QAM } \\
(\mathbf{d B})\end{array}$ \\
\hline IFDMA & --- & --- & 3.8 & 5.0 \\
\hline LFDMA & 7.9 & 7.9 & 8.3 & 8.3 \\
\hline OFDMA & 11.9 & 11.1 & 11.3 & 11.5 \\
\hline
\end{tabular}

Now, let us see the performance of IFDMA,LFDMA and OFDMA in the presence of Raised Cosine filter. RC filter is generally used for the pulse shaping. Next result shows the performance of differnet subcarrier allocation by varying the value of $\mathrm{RC}$ filter, for these simulation we took values of $\mathrm{RC}$ filter in range between 0 to 1 , with a spacing of 0.2 , so after every 0.2 spacing we could analyze the perfromance of such techniques in presence of DFT-Spreading code. for this simulation we have taken only two modulation techniques in our account QPSK and 16-QAM. 


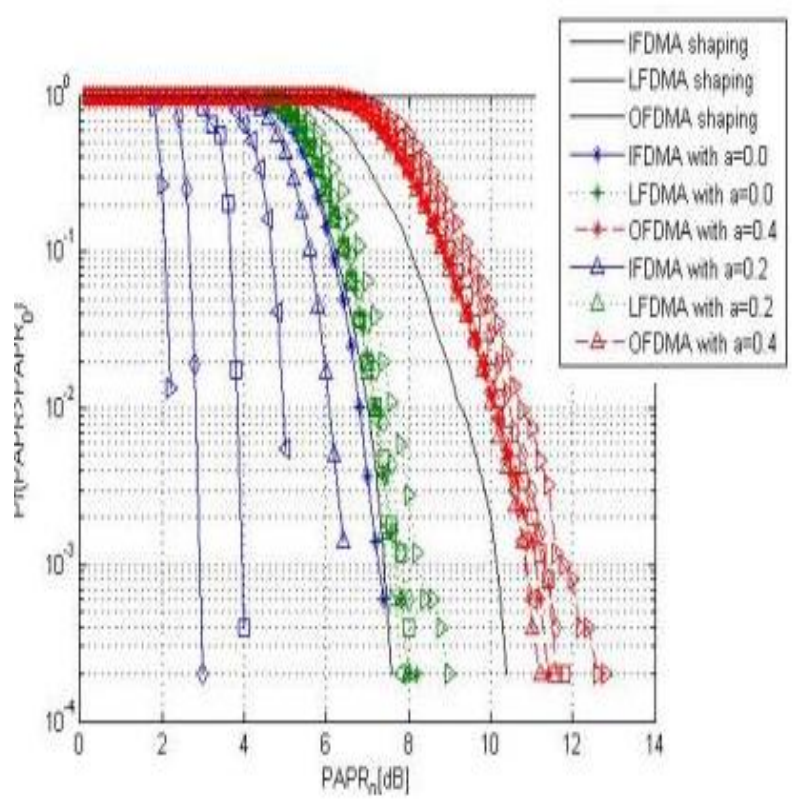

Fig 9: Performance of different allocation method with QPSK and different values of pulse shaping filter.

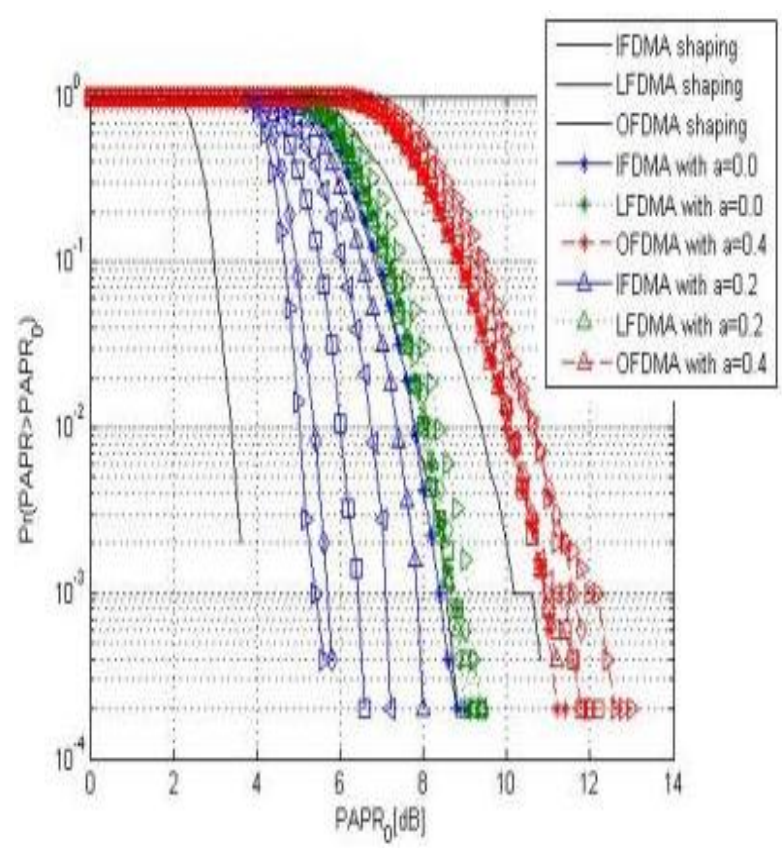

Fig 10: Performance of different allocation method with 16-QAM and different values of pulse shaping filter.

So on the basis of fig 9 , fig 10 we can easily justify that the performance of 16-QAM is better at every value of RC pulse shaping filter and without pulse shaping as well.

Now in next graph we analyzed that by increasing the value of spreading code on IFDMA, LFDMA, subcarrirer allocation method with a specific 0.4 value of pulse shaping.

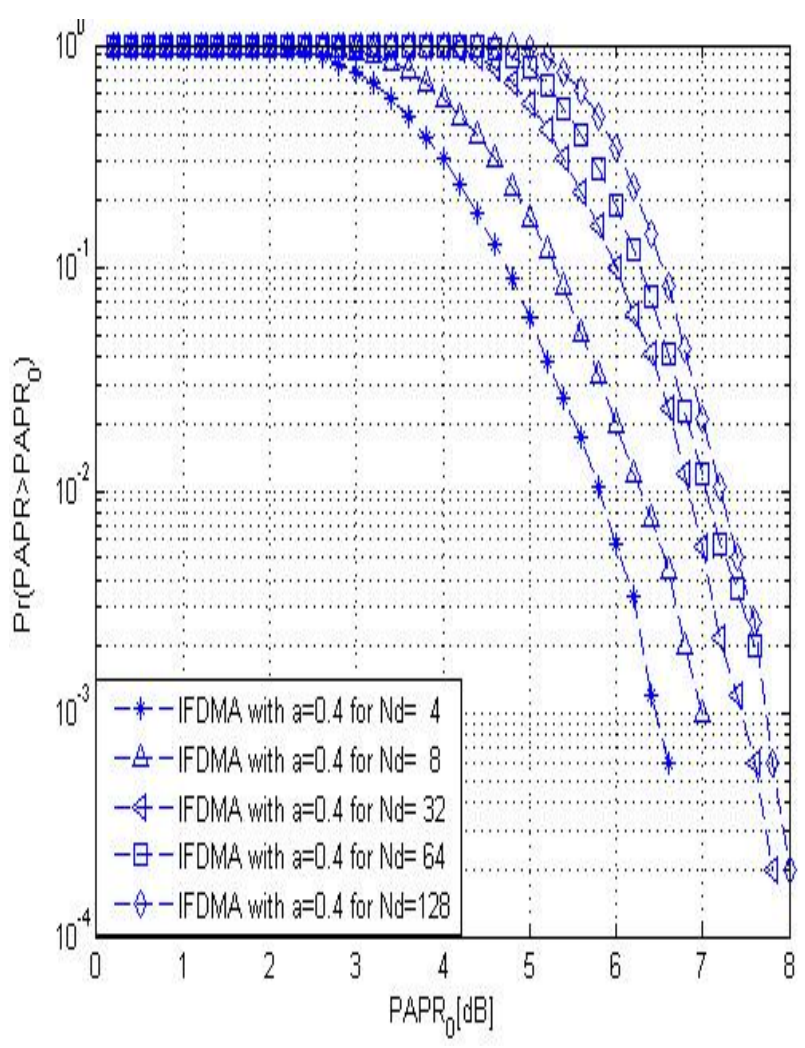

Fig 11: Performance of IFDMA with different values of spreading code and value of pulse shaping

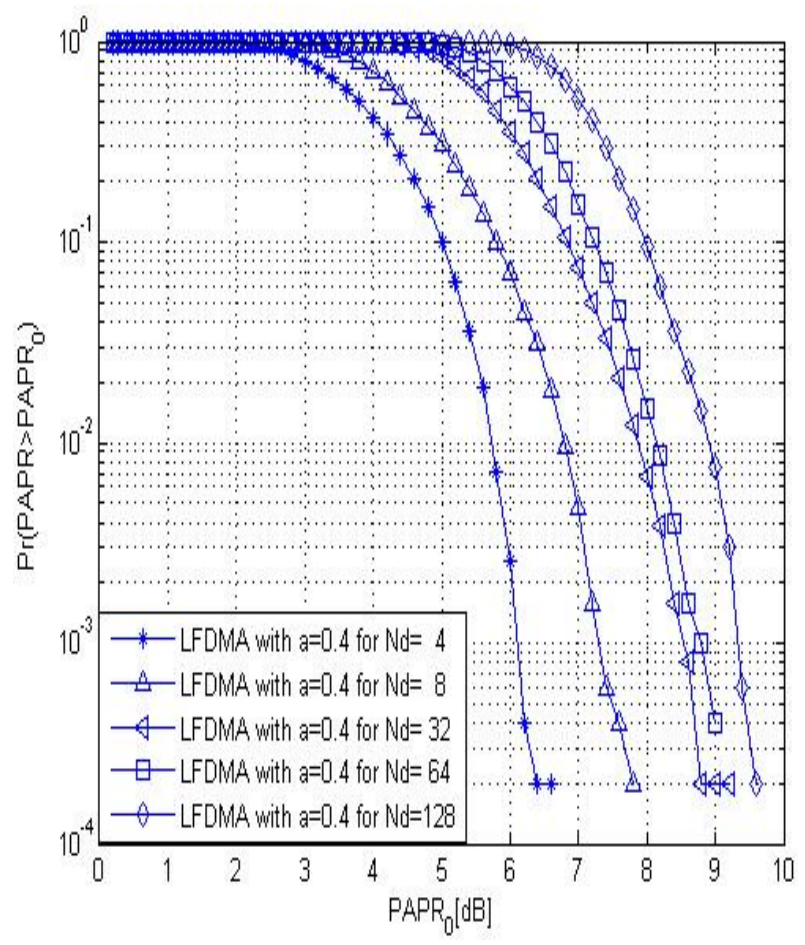

Fig 12: Performance of LFDMA with different values of spreading code and value of pulse shaping. 


\section{CONCLUSION}

On the basis of above result we can conclude that by using DFT- spreading technique, with different subcarrier allocation method, the performance of IFDMA system is better as compare to the other allocation methods, such as LFDMA and OFDMA prescribed in table 1 . When we introduce raised cosine filter with value of 0 to 1 with a spacing of 0.2 , the performance of 16-QAM and IFDMA is better with other two allocation methods and QPSK, and in the last section we used different DFT-spreading code values vary from 4 to 128 on IFDMA and LFDMA technique fig.11, fig.12 shows the minimum spreading code gives maximum results, so the lower value of DFT-spreading code is suitable for higher performance.

\section{FUTURE SCOPE}

As we know from the above results the performance of IFDMA technique is better than the LFDMA and OFDMA technique, but specially if we talk about DFT spreading allocation methods, then the implementation of IFDMA technique is more complex as compare to the LFDMA technique, because IFDMA needs interleaved data sequences which is difficult to maintain and needs pilots insertion, so LFDMA is more popular as compare to the IFDMA, so by inserting pilots we can implement IFDMA technique more easily.

\section{ACKNOWLEDMENT}

First and foremost we would like to thank our Research guide Sr. Assistant Prof. Mrs. Ratna Gour, We would also like to thank Assistant Prof. Mr. Abhijeet Bishnu \& Assistant Prof. Anshul Shrotriya for their valuable guidance.

\section{REFERENCES}

[1] Y. Wu and W. Y. Zou, "Orthogonal frequency division multiplexing: A multi-carrier modulation scheme, "IEEE
Transaction. Consumer Electronics, vol. 41, no. 3, pp. 392-399, Aug. 1995.Ding, W. and Marchionini, G. 1997 A Study on Video Browsing Strategies. Technical Report. University of Maryland at College Park.

[2] Manushree Bhardwaj, Arun Gangwar., "An Overview: Peak to Average Power Ratio in OFDM system \& its Effect",IJCCTS Volume 01 - No.2, Issue: 02 September 2012.

[3] Bader HamadAlhasson, Mohammad A. Matin, "PAPR Performance analysis of DFT-spread OFDM for LTE Uplink transmission" IJCSIS Volume 09 - No.10, October 2011.

[4] Vendee-Ramjee, "OFDM for wireless multimedia communications".

[5] R. Kimura and F. Adachi, "Comparison of OFDM and multicode MC-CDMA in a frequency selective fading channel, "IEE Electronics Letters, vol. 39, no.3, pp. 317 318, Feb. 2003

[6] Gillian Huang, Andrew Nix, Simon Armour, "Impact of radio resource allocation and pulse shaping on PAPR of SC-FDMA signal, "The 18th Annual IEEE International Symposium on Personal, Indoor and Mobile Radio Communications2007.

[7] M. M. Rana, Md. Saiful Islam and Abbas Z. Kouzani, "Peak to Average Power Ratio Analysis for LTE Systems", second International Conference on Communication Software and Networks 2010.

[8] Yuan Fang, Li Tao and Nan Chi, "Interleaved subcarrier allocation for DFT-spread OFDM to reduce PAPR", " $17^{\text {th }}$ opto electronics and communication conference (OECC 2012), july 2012, buasn korea." 